Revue de l'Institut des langues et cultures

d'Europe, Amérique, Afrique, Asie et Australie

19 | 2014

Acceptabilité et transgression en langues et cultures de spécialité

\title{
"Thinking outside of the box » : réflexion sur l'acceptabilité en anglais lingua franca professionnelle
}

Thinking Outside of the Box: Perspectives on Acceptability in English as a Professional Lingua Franca

Philippe Millot

\section{OpenEdition}

Journals

Édition électronique

URL : http://journals.openedition.org/ilcea/2499

DOI : $10.4000 /$ ilcea.2499

ISSN : 2101-0609

Éditeur

UGA Éditions/Université Grenoble Alpes

Édition imprimée

ISBN : 978-2-84310-280-6

ISSN : $1639-6073$

Référence électronique

Philippe Millot, « «Thinking outside of the box » : réflexion sur l'acceptabilité en anglais lingua franca professionnelle », ILCEA [En ligne], 19 | 2014, mis en ligne le 27 juin 2014, consulté le 20 avril 2019. URL : http://journals.openedition.org/ilcea/2499 ; DOI : 10.4000/ilcea.2499

Ce document a été généré automatiquement le 20 avril 2019

(C) ILCEA 


\title{
«Thinking outside of the box »: réflexion sur l'acceptabilité en anglais lingua franca professionnelle
}

\author{
Thinking Outside of the Box: Perspectives on Acceptability in English as a \\ Professional Lingua Franca
}

Philippe Millot

1 La notion d'anglais lingua franca et, plus particulièrement, celle de l'anglais comme lingua franca professionnelle repose sur l'observation suivante : un nombre très important de professionnels utilisent une variété de langue qui, bien qu'elle ne respecte pas la totalité des normes de l'anglais britannique ou américain, généralement désignée sous le terme d'anglais «standard» (Trudgill \& Hannah, 2008), leur permet d'obtenir le niveau d'efficacité suffisant pour atteindre leur objectifs communicatifs. L'enquête de terrain que nous avons menée, ainsi que les autres études réalisées dans ce domaine (Nickerson, 2000; Kankaanranta, 2005), montrent que le degré d'acceptabilité en anglais professionnel ne se mesure pas forcément en fonction des écarts par rapport à la norme de l'anglais standard, mais en fonction de critères plus globaux qui affectent l'efficacité de la communication dans son ensemble. Cette manière d'envisager la norme linguistique n'est pas nouvelle. Elle s'inscrit dans la longue tradition des études de la variation et des grammaires fondées sur l'observation des usages (Fries, 1940 ; Labov, 1972 ; Milroy, 1987 ; Biber et al., 1999). Dans ce qui est souvent présenté comme la première grammaire américaine sur corpus (Fries, 1940), l'auteur indique que l'acceptabilité ne saurait être définie par la simple opposition entre un « usage général » et des « erreurs » par rapport à cet usage. L'acceptabilité en langue dépendrait au contraire d'un ensemble complexe de critères historiques et statistiques conduisant certains groupes sociaux à accepter ou rejeter un usage. L'auteur observe, par exemple, que dans la classe populaire américaine, l'absence de la marque du pluriel dans les unités de mesures (voir exemple 1) est déviante par rapport à l'usage dit « général ». Cependant, par son caractère systématique et peu gênant pour la communication, cette absence de marque peut être considérée comme acceptable, tant d'un point de vue social que scientifique. 
Exemple 1. Extrait de la grammaire de Fries (1940, p. 43) sur les usages des classes populaires américaines.

about five foot away

from 5 to 10 gallon of water

havn't herd from him for two month

2 À travers sa description fondée sur des réalisations linguistique réelles, il démontre ainsi que cette population possède, en quelque sorte, sa propre grammaire. Ce qui définit le seuil d'acceptabilité n'est donc pas l'utilisation de telle ou telle forme en soi, mais la conformité de l'utilisation à la situation que Charles $\mathrm{C}$. Fries définit à partir de catégories sociales. Dans cet article portant sur l'acceptabilité dans le cadre particulier des échanges professionnels, nous définissons la situation comme l'ensemble des paramètres régulant les usages au sein d'un réseau socio-professionnel de participants engagés dans la réalisation d'actions conjointes.

Dans ce cadre variationniste, l'anglais comme lingua franca, c'est-à-dire l'anglais comme langue d'échange entre non-natifs, pose toutefois un problème particulier. Il s'avère, en effet, que les études variationnistes concernent, dans leur très grande majorité, des locuteurs natifs, c'est-à-dire des locuteurs dont la légitimité à parler cette langue et à la faire varier, est incontestable. Dans la situation lingua franca, la légitimité est plus douteuse, car les locuteurs non natifs sont traditionnellement considérés comme des apprenants, c'est-à-dire des locuteurs dont l'identité est directement liée à la notion d'écart par rapport à la norme à atteindre. Les locuteurs non natifs de l'anglais sont donc définis comme des locuteurs potentiellement enclins à produire des énoncés anormaux, ce qui tend à leur conférer un statut de locuteurs illégitimes.

\section{L'anglais lingua franca : une variété en voie d'acception}

Les «cercles» de Braj Kachru (1985), représentés dans la figure 1, ont largement contribué, et contribuent encore aujourd'hui, à la représentation du monde anglophone dans les travaux scientifiques, ainsi que les ouvrages et articles visant un plus large public (Graddol, 2000 ; Crystal, 2003). En plaçant l'anglais britannique et américain au centre de la carte, B. Kachru présente les variétés natives comme le point focal de l'anglais dans le monde. Cette place sur la carte résulte bien évidemment d'une histoire linguistique où les îles britanniques, puis les États-Unis d'Amérique, peuvent être considérés comme le berceau de l'anglais tel que nous le connaissons aujourd'hui. Cette place sur la carte fournit, par ailleurs, une réponse au besoin de stabilité normative pour une langue dont les enjeux culturels, politiques et économiques au niveau international sont considérables. La variété « anglais international » est par exemple définie par P. Trudgill et J. Hannah (2008) sur la base des variétés natives qui, selon les auteurs, présentent l'avantage d'avoir été « codifiées et stabilisées » (p. 1). 
Figure 1. - Les cercles de B. Kachru (1985) dans D. Crystal (2003, p. 61).

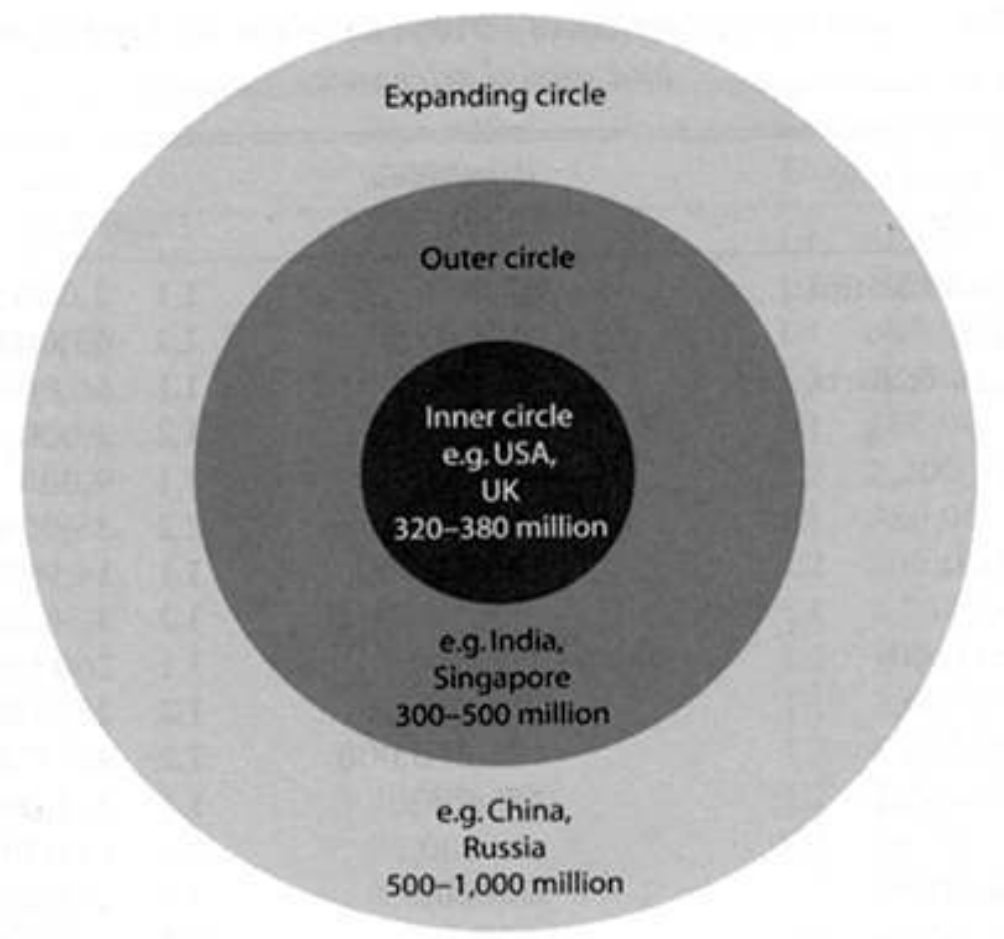

Cette représentation contribue à la forte valorisation du cercle des locuteurs natifs par les membres des cercles périphériques, notamment les enseignants d'anglais comme langue étrangère. Sur le plan phonétique par exemple, Jennifer Jenkins (2007) démontre que la Received Pronunciation, pourtant peu répandue dans les îles britanniques, conserve une forte puissance normative. Dans son enquête de terrain, les 326 enseignants interrogés et issus des trois cercles kachruviens expriment, en effet, une nette préférence pour les accents du cercle intérieur, les accents non natifs étant considérés comme "peu corrects » (p. 179).

6 Toutefois, du fait des apports de la sociolinguistique de la variation, de la linguistique de corpus et l'émergence du domaine des World Englishes, l'ethnocentrisme anglo-américain est progressivement remis en cause. Certains chercheurs comme Gerhard Leitner (1992) soulignent le fait que, d'un point de vue statistique, les locuteurs natifs forment désormais une minorité, la plupart des locuteurs se situant dans les cercles périphériques. L'auteur propose, par exemple, de sortir des cercles kachruviens et dessine une nouvelle carte mentale du monde anglophone, composée d'un « anglais du Nord», dont la norme se rapproche des variétés britannique et américaine, et d'un "anglais du Sud» regroupant les variétés d'Afrique et d'Asie. Cette volonté de sortir du paradigme habituel s'exprime également à travers l'identification de six grandes variétés régionales de l'anglais par Yasukata Yano (2009) que sont les anglais asiatique, arabe, européen, africain, latin et anglo-américain, l'anglais européen et asiatique faisant actuellement l'objet d'une recherche particulièrement nourrie (Jenkins et al., 2001; Kirkpatrick, 2012 ; Galloway \& Rose, 2013 ; Wang, 2013). En filigrane, ces travaux suggèrent que les critères géographiques pour définir une norme de l'anglais comme langue internationale sont relativement peu pertinents. Nous suggérons donc ici de sortir du paradigme géo-centré pour rejoindre celui des communautés mondialisées qui, à travers l'atteinte d'objectifs 
professionnels (Kankaaranta, 2005) ou universitaires (Mauranen et al., 2010), recourent au code source de l'anglais, pour ensuite y inclure des éléments propres à leur identité.

Dans cette perspective, le locuteur modèle n'est plus nécessairement le locuteur natif mais l'utilisateur expérimenté de la langue anglaise qui, quelle que soit sa nationalité, est capable de faire face à la multiplicité des situations d'échange. Dans ce cadre protéiforme, le «bon comportement ", selon les précurseurs de la variété English as a Lingua Franca (Barbara Seidlhofer, Henry Widdowson, Jennifer Jenkins et Anna Mauranen), n'est pas nécessairement le respect de la norme anglo-américaine, mais la capacité des locuteurs à puiser dans les ressources linguistiques dont ils disposent en vue de produire des messages accessibles et acceptables en fonction de la situation d'échange. En d'autres termes, nous envisageons l'anglais comme lingua franca non pas comme une somme d'écarts par rapport à une norme native dans le cadre d'un apprentissage, mais comme un phénomène en tant que tel, une variété légitime en ce qu'elle répond à un besoin de communication de millions d'utilisateurs, non natifs, non apprenants à travers le monde. Comme nous allons le voir, le phénomène trouve chez les professionnels un terrain de réalisation et d'observation tout à fait fertile permettant de dégager des critères d'acceptabilité dans ce domaine.

\section{Paradigme du professionnel-utilisateur « global » de la langue anglaise}

En France, chez les professionnels travaillant dans des contextes internationaux comme les employés des multinationales, l'anglais est devenu, en quelques dizaines d'années, une lingua franca professionnelle que nous définissons comme une langue de travail que les professionnels utilisent lorsqu'aucune autre langue commune n'est disponible. L'enquête de terrain que nous avons menée sur l'utilisation des courriers électroniques en anglais montre que cette variété de langue émerge dans des situations spécifiques et éphémères (le temps de résoudre un problème ou d'effectuer une mission) et au sein de réseaux épistolaires de relativement petite taille, généralement très spécialisés. Ce type d'échange, que la technologie du courrier électronique favorise largement, conduit à un accroissement considérable de la communication en anglais dans les entreprises. À cet accroissement en volume, il convient, toutefois, d'ajouter un accroissement de la diversité des interlocuteurs de cultures professionnelles différentes, notamment la «culture de fonction » telle que la « culture de cadre » ou la « culture de secrétaire » (Isani, 2004). Sur ce point, la fonction de manager dans le contexte des entreprises multinationales peut être présentée comme l'archétype du phénomène d'internationalisation des fonctions et des métiers dans l'entreprise multinationale. Le témoignage suivant, issu de notre enquête de terrain, illustre clairement le phénomène. Il souligne le caractère spécialisé de l'anglais comme lingua franca professionnelle au sens où cette variété de langue émerge dans le cadre d'actions (ici la conception, la distribution de produits, la coordination ou encore le marketing) qui requièrent des compétences relevant de cultures professionnelles spécifiques.

J'appartiens à une Business Unit (sic !) du groupe [...]. Cette business unit s'appelle [...]. Elle conçoit et distribue des accessoires informatiques. J'en suis le directeur régional, en charge de plusieurs pays européens (France, Espagne, Portugal, Italie, Allemagne, Suisse Autriche). À ce titre, je dirige et coordonne l'ensemble des activités de [...] : ventes, marketing, logistique, finances, ressources humaines, etc. 
Parce que cette variété est ancrée dans une problématique de terrain, à la fois mondiale et spécialisée, le bon usage ne saurait être calqué sur celui d'un locuteur natif, mais sur celui de l'utilisateur «global » et expérimenté, confronté à la "super-diversité » (Cogo, 2012) des réseaux socio-professionnels. Par utilisateur "global» et expérimenté, nous entendons un employé qui, par sa pratique professionnelle, parvient à un niveau de maîtrise suffisant pour faire face à la diversité des situations professionnelles. Le manager que nous venons de présenter et dont le réseau épistolaire électronique s'étend sur 5 pays différents au moment de notre observation (voir fig. 2), peut être considéré comme un exemple représentatif de millions d'utilisateurs de l'anglais comme lingua franca professionnelle dans le monde.

Figure 2. - Réseau socio-professionnel d'un manager représenté à partir d'échanges de courriers électroniques.

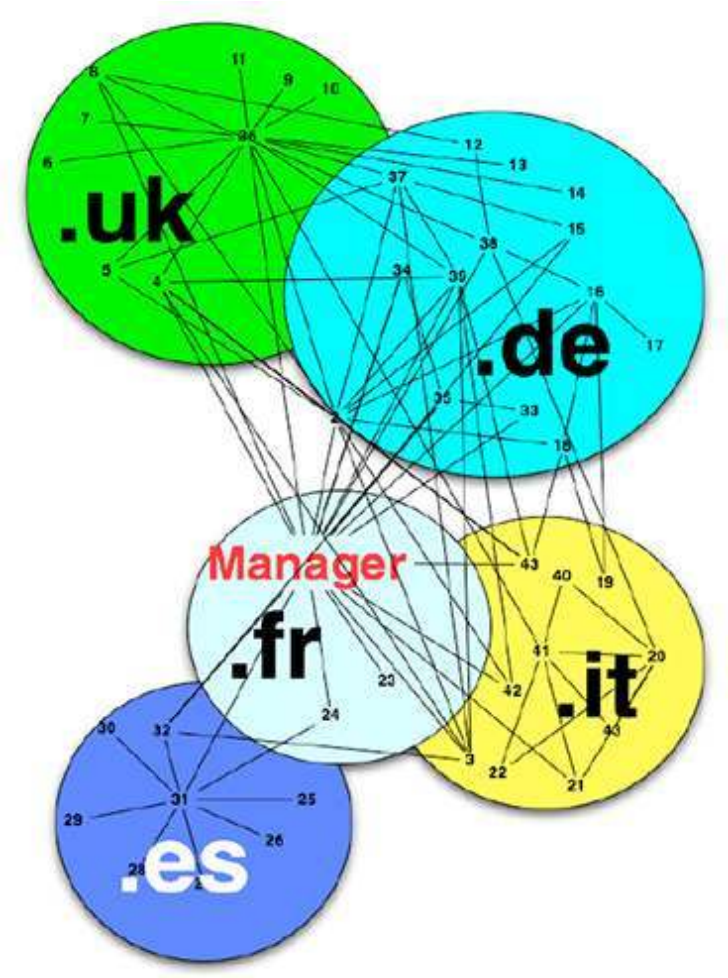

Cette représentation implique, selon nous, une certaine remise en cause du statut de locuteur natif comme seul point de repère normatif dans les situations d'échanges internationaux. Nous présentons ci-dessous un nouveau modèle qui permet de rendre compte de nos observations. Comme la figure 3 l'indique, ce modèle se fonde sur les actions professionnelles qui, comme l'exemple précédent l'a montré, forment la raison d'être de la plupart des échanges. Ces actions, réalisées au sein de réseaux socioprofessionnels internationaux, constituent les situations d'échange, chaque situation pouvant être définie à partir de divers paramètres tels que le statut de l'anglais chez les locuteurs, le type d'action, le domaine professionnel. L'ensemble de ces paramètres, dont les combinaisons sont potientiellement très nombreuses, conduisent enfin à des utilisations de l'anglais dont les critères d'acceptabilité dépendent directement du paramètrage de la situation d'échange. 
Figure 3. - Modèle utilisateur « global » de l'anglais comme lingua franca professionnelle.

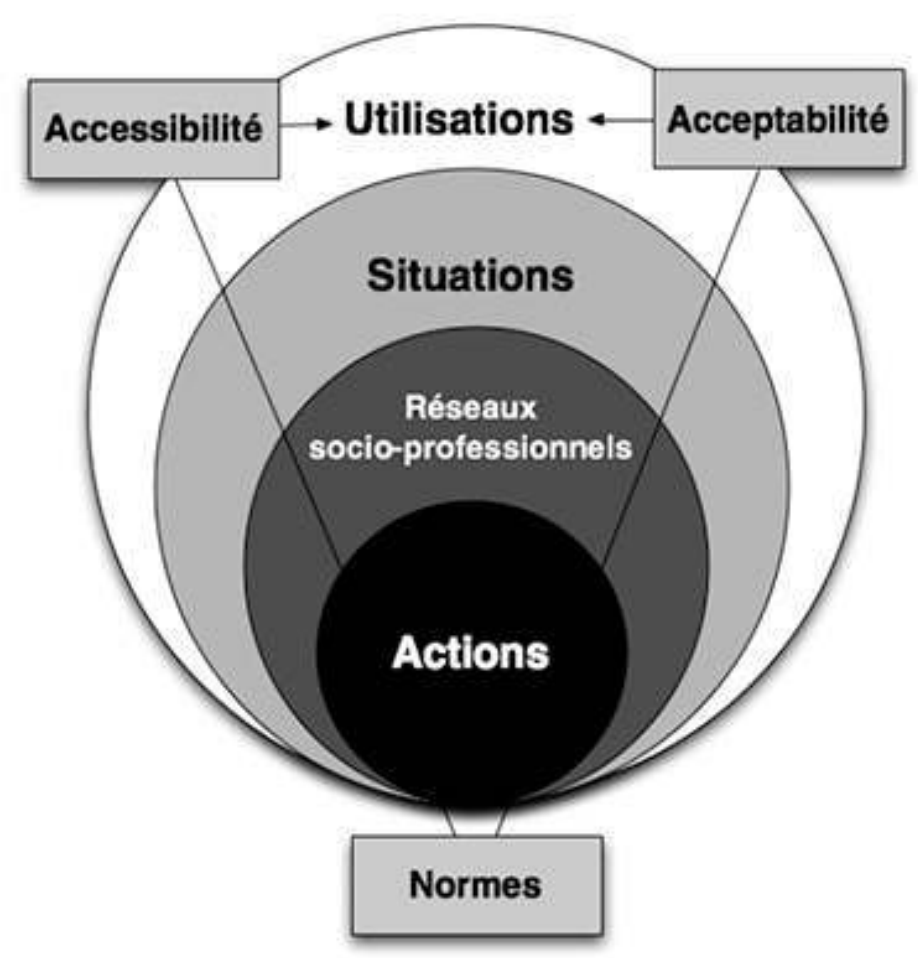

Dans ce cadre dynamique, la frontière entre l'acceptable et l'inacceptable tend à varier fortement pour trois raisons essentielles. La première est que, comme pour toute variété de langue, le degré d'acceptabilité dépend fortement des situations d'échange. Ce qui est par exemple considéré comme une erreur dans une situation d'échange entre natifs ne le sera pas nécessairement dans une situation entre non-natifs où le primat est donné à l'accessibilité du message. La deuxième raison est que l'attitude face à une utilisation dépend davantage de la conformité de la contribution à la réalisation de l'action qu'au respect du code de la langue. À cela, il convient d'ajouter la troisième raison du besoin de maintenir la cordialité entre les acteurs sociaux afin de ne pas entraver la réalisation des actions. Comme nous allons le voir, ce besoin de cordialité conduit les professionnels à « laisser passer » les écarts par rapport à l'anglais standard.

Ce relativisme normatif, pour ne pas dire cette fluidité normative, n'est pas sans poser quelques problèmes à la fois théoriques et pratiques, notamment celui du besoin de norme commune chez les professionnels engagés dans des situations d'échanges protéiformes. Face à ce problème, nous proposons trois critères qui, d'après nos observations, contribuent au succès des échanges et, par là-même, à leur caractère acceptable en dépit des écarts par rapport à l'anglais dit « standard ». 


\section{Critères d'acceptabilité en anglais comme lingua franca professionnelle}

\section{La conformité au genre}

13 Le corpus de 400 courriels que nous avons constitué au cours de notre enquête de terrain montre que la correspondance électronique fait intégralement partie de la culture professionnelle des employés. Cette correspondance contribue en effet à la réalisation du genre de la « résolution de problèmes », qu'Almut Koester (2010) décrit comme le genre le plus répandu de la vie en entreprise. Nous considérerons ici la résolution de problème comme un " macro-genre " générant des séquences discursives réalisées sur mode écrit ou oral. L'omniprésence des courriels dans ces séquences révèle que ce moyen de communication sert à remplir plusieurs buts communicatifs. Il permet tout d'abord de transmettre et d'archiver les données « solides » telles que des données chiffrées et toute autre information précise sur les situations en cours, ce qui correspond au « genre de la lettre postale ${ }^{1}$ » identifiée par Anna Kankaanranta (2005). Il permet ensuite l'échange « fluide » d'informations et de points de vue, ce que l'auteur nomme le genre " dialogue ».

L'analyse de nos données montre clairement que le genre est systématiquement conforme à la situation, les variations constatées ayant des objectifs stratégiques et interpersonnels précis. Le caractère systématique de la conformité du genre à la situation fait du genre un critère puissant d'acceptabilité des messages, une infraction aux caractéristiques les plus centrales pouvant paraître curieuse et rendre le message suspect. Parmi ces caractéristiques centrales, nous pouvons noter la présence d'un objet de message, d'une signature et d'un corps de message, les autres parties structurantes telles que les formules de salutation pouvant faire l'objet de variations fréquentes en fonction de la situation. Nous notons également que le respect des normes typographiques propres au genre, c'est-à-dire le fait d'écrire en casse normale et non en lettres capitales, font partie des règles à suivre. Dans l'exemple suivant du genre « dialogue », tiré d'un échange entre un manager français et son équipe internationale, l'écriture en lettres capitales et en corps gras (message 1) est perçue comme curieuse et fait l'objet d'un commentaire (message 3) :

Exemple 2. Humour et informalité dans un échange de courriels entre managers.

Message 1 [L1 = NL] : GIVEN THE REMARK THAT YOU WANTED TO GO TO

AUSTRALIA BY THE END OF JULY 2007, I SUGGEST THAT YOU WILL COME TO

KOREA AFTER THAT TRIP. OK?

Message 2 [L1 = Ang (Singapour)] : How? go? Cheers.

Message 3 [L1=FR] : I guess this will be Korea then! Guys that write in BOLD

CAPITAL LETTERS are normally not easy to deal with!!

Le message 3, si informel soit-il, peut être considéré comme tout à fait acceptable et ne saurait constituer une erreur de style ou un manque de professionnalisme. Selon A. Koester en effet, l'informalité et l'humour forment une caractéristique essentielle des échanges en milieu professionnel.

Outre les dialogues par courriel générant régulièrement une certaine informalité, les situations d'échange de données plus formelles, comme les données commerciales, techniques ou administratives, tendent à générer des micro-genres davantage conformes aux canons épistolaires. Le message qui suit est un exemple typique où le respect scrupuleux des canons épistolaires tels que nous pouvons les rencontrer dans les 
échanges de courriels contribue à masquer, en quelque sorte, les erreurs par rapport à l'anglais standard. En dépit des problèmes, le message prend donc un caractère «normal » et acceptable dans son contexte d'échanges entre non-natifs pour qui l'accès à l'information l'emporte sur la correction linguistique. L'accès à l'information est tout d'abord facilité par des stratégies linguistiques comme l'utilisation de vocabulaire lié à la situation (stickers, print, database, whirpool stickers, plant), le vocabulaire lié au genre (hello, regards) et la répétition lexicale (stickers, sent) pour limiter l'ambiguïté. Il est ensuite facilité graphiquement par la séparation des différents éléments de l'information à l'aide du saut de ligne permettant de les mettre en valeur. Au-delà des problèmes de langue, que tout angliciste ne manquera pas de constater (problèmes récurrents dans la détermination des noms, traduction approximative de «bien installer », et l'orthographe même du mot «base de données »), l'efficacité reste relativement intacte au sens où les participants parviennent à échanger des informations solides.

Exemple 3. Stratégies d'efficacité dans la réalisation du genre. Message envoyé par une chef de projet française d'une entreprise de fabrication de salles de bain à une collaboratrice russophone travaillant dans une usine près de Moscou.

Hello,

Last data base was sent to China with final stickers format.

China will sent to me some stickers to validate that they have got well install the system in their plant.

[placeName01] is ready to print stickers.

Concerning whirlpool stickers, [placeName01] need to provide to [placeName02]

(whirlpool system will be install in [placeName02]) some stickers.

regards

[forename14]

Nous retrouvons ce type de caractéristiques, où les stratégies d'efficacité priment sur la correction de la langue stricto sensu, dans d'autres domaines. Dans l'exemple ci-dessous, tiré d'un échange entre ingénieurs réalisant des tests sur un nouveau modèle automobile, les stratégies sont similaires à l'exemple précédent. En dépit des rares écarts par rapport au code de l'anglais standard, le vocabulaire de la situation (vehicle speed signal, noise, reported noise, level, evaluated) est maîtrisé et la répétition de certains éléments (« vehicle », «noise », «speed signal ») vient limiter l'ambiguïté. Par ailleurs, les éléments structurant l'information sont mis en évidence graphiquement et phraséologiquement, chaque paragraphe réalisant un objectif communicatif spécifique, lequel objectif est signalé par le biais d'une phraséologie explicite ("can you inform me», "I would like to know»). L'ensemble de ces stratégies, dont la maîtrise du code ne forme qu'un aspect, contribue à une cohérence d'ensemble, rendant le message efficace dans son contexte.

Exemple 4. Stratégies d'efficacité dans la réalisation du genre. Message d'un ingénieur français à un partenaire japonais.

Object : [ref00] question about ' $\mathrm{Pb}$ de stabilité en [ref01]'

Hello [forename233],

can you inform me if the reported noise on the vehicle speed signal can be found on any vehicle in the project [refoo]? The reason why I ask is that we couldn't find any such noise on the car we keep as reference in [placeName]: [ref01].

I would like to know this to be sure that we know the rootcause of the noise on the vehicle speed signal. (How are we sure the current reported noise level is stable?) We evaluated altough the [ref01] with the proposed calibration changes and we could not find any negative influence on CC performance.

Best regards

[forename234] 
17 En conclusion, contrairement aux situations natives où les connaissances partagées sont relativement homogènes, les connaissances partagées dans les situations lingua franca sont, par nature, hétérogènes et possèdent, de manière inhérente, une part d'inconnu. Il s'ensuit que les utilisateurs de l'anglais lingua franca recourent, de manière nettement plus marquée que dans les situations natives, aux stratégies de maintien de la cordialité et de clarté évoquées plus haut. Quel que soit le degré d'adresse avec lequel ces stratégies sont réalisées, elles contribuent à la bonne réalisation des actions et des genres.

\section{Le respect des logiques professionnelles}

Parce que ces types de problèmes relèvent de domaines professionnels différents, ils engagent des professionnels qui, par leur formation et leurs métiers, sont amenés à rédiger des courriels dans des styles professionnels tout aussi différents, chaque style étant considéré comme normal et acceptable dans son contexte. Dans le cadre de cet article, nous réduirons la notion de style professionnel au fait que les rédacteurs de message mettent en œuvre des logiques professionnelles spécifiques que Françoise Mourlhon-Dallies (2008) définit comme des routines discursives représentatives de la manière dont les professionnels pensent et réalisent leur métier. Dans les échanges de courriers électroniques, les logiques se présentent sous la forme typique d'un style binaire alternant objectivité (constatation, rappel des faits, etc.) et subjectivité (attitude face à la situation). En anglais lingua franca, l'alternance est particulièrement marquée et présente une séquence relativement régulière où chaque proposition ou complexe propositionnel est, en quelque sorte « spécialisé » dans la réalisation de chaque partie de la logique.

19 L'exemple ci-dessous, tiré d'échanges entre ingénieurs électroniciens français et danois, présente le cas de ce que nous appelons la logique du rapport d'erreur. Cette logique consiste en la présentation d'un fait (« No pumping effect, the behaviour of the Gap Opt is quite $O K$, except if two bins arrived with a gap of approximatly $80 \mathrm{~cm} »)$, présenté comme relativement objectif, suivie d'une évaluation de la situation par le professionnel («Is looks like the speed is not correctly choosen and the exit gap stay to $40 \mathrm{~cm} »)$.

Exemple 5. Logique du rapport d'erreur entre ingénieurs électroniciens français et danois (secteur électronique grand public).

E22: No pumping effect, the behaviour of the Gap Opt is quite OK, except if two bins arrived with a gap of approximatly $80 \mathrm{~cm}$, Is looks like the speed is not correctly choosen and the exit gap stay to $40 \mathrm{~cm}$.

20 L'exemple suivant présente une logique similaire où l'ingénieur commence par poser le problème (« issue ») pour ensuite inviter son interlocuteur à l'action (« action required »).

Exemple 6. Logique du rapport d'erreur entre ingénieurs électroniciens français et japonais (secteur automobile).

Issue:

indicator accuracy doesn't meet with [orgNameG02] testing criteria. Error rate should be between $+5 \%$ and $-8 \%$. The testing value is approx $+18 \%$.

Action required:

If you have any reason why the error rate is so high, Please show us the reason. (possibly [refoo] data mistake?) Would you show us any countermeasure idea to fix this problem.

21 Le style binaire des logiques professionnelles émerge également dans des domaines moins techniques comme celui des ressources humaines où le rappel de la procédure (présentée 
comme un fait objectif, en gras dans l'exemple) alterne avec l'évaluation de la situation (en caractère normal). Dans l'exemple ci-dessous, un assistant RH informe diplomatiquement un manager qu'il sera impossible de former une nouvelle employée dans les conditions demandées et rappelle objectivement la procédure concernant les délais de formation.

Exemple 7. Logique administrative dans des ressources humaines.

I am not sure whether you could put [forename407] in charge of doing some courses in order to reduce or split your schedule. Just for your information, according to our procedure [refoo] induction trainings must be done within 2 weeks following the 1st day of employment. [forename405] will join [orgNameA0] on July 2nd 2007 and all induction trainings must be done on July 13 th at the latest.

La fréquence élevée de ces logiques dans notre corpus suggère qu'elles font partie, avec le genre, des connaissances partagées entre interlocuteurs de langue et cultures différentes. Tout comme pour le genre, la réussite de leur réalisation paraît relativement indépendante du niveau de maîtrise de la langue. Dans l'exemple ci-dessous, une partie de la logique de la requête présente des erreurs pouvant être considérées comme basiques par rapport à l'anglais standard (redondance de could et be able, orthographe incorrecte de « abble»).

Exemple 8. Logique de la requête entre managers français et chinois.

Different kind of laser grave pattern will need to be tested: points, Lines, Could you

be abble to make that tests for us?

Nous considérons, cependant, ce message comme globalement réussi dans la mesure où les erreurs ne feront l'objet d'aucun commentaire de la part de l'interlocutrice dont la préoccupation n'est pas la correction de la langue en soi, mais l'accès à l'information. La réussite du message repose également sur la réalisation d'une requête à partir d'un schéma habituel d'alternance entre la présentation d'un contexte formulé de manière objective et la requête proprement dite. Le caractère habituel est renforcé par l'amorce " Could you » qui, parce qu'elle relève d'une phraséologie récurrente dans l'expression des requêtes, fonctionne tel un signal permettant à l'interlocuteur d'identifier immédiatement la fonction communicative de la proposition «be abble to make that tests for us ».

En conclusion, nous observons que les logiques professionnelles fonctionnent tel un filtre supplémentaire par rapport au genre, garantissant, en quelque sorte, l'accessibilité des messages. Dans ce cadre, nous suggérons que la réussite des messages provient d'une interaction entre le niveau global de la réalisation du genre et le niveau plus local de celle des logiques professionnelles où certains schémas phraséologiques jouent un rôle d'indicateur de fonction communicative. Les niveaux du genre et de la logique étant généralement partagés par les professionnels de fonctions similaires, les messages fortement erronés par rapport au code de l'anglais standard ne suscitent pas de réactions particulières, les participants préférant «laisser passer » pour ne pas entraver le bon déroulement de l'action en cours. Dans ce cadre, le code de l'anglais apparaît comme le dernier niveau. Il s'apparente davantage à un code « open source ", c'est-à-dire malléable, à la fois similaire au code d'origine dont il tient sa source, et ouvert dans la mesure où des natifs d'autres langues que l'anglais se le sont approprié et l'ajustent en fonction de leurs besoins. 


\section{Malléabilité du code de l'anglais}

Par tradition et par une «certaine facilité " (Widdowson, 2012), les critères d'acceptabilité du code de l'anglais reposent sur la conception chomskyenne de la compétence en langue. Cette conception repose sur le concept de «locuteur-allocutaire idéal » que Chomsky définit de la façon suivante: «un locuteur-allocutaire dans une communauté de parole complètement homogène et qui connaît sa langue parfaitement ${ }^{2}$ " (Chomsky, 1965, p. 3). Quelques années plus tard, le concept est repris par Hymes sous l'appellation de locuteur «normal» que l'auteur définit de manière relativement intuitive : «Il relève du bon sens qu'un membre normal d'une communauté connaisse les systèmes de communication qui s'offrent à lui ${ }^{3}$.» (Hymes, 1972, p. 282) Selon l'auteur, ce "membre normal », un natif en réalité, serait le seul apte à formuler des jugements d'acceptabilité sur le caractère grammatical, faisable, approprié au contexte et conforme à la pratique habituelle. Bien que cette conception soit relativement ancienne et qu'elle ait fait l'objet d'une critique approfondie encore très récemment (Seidlhofer, 2011; Shaw, 2011), force est de constater que le concept de «locuteur-allocutaire idéal» ou de « membre normal » d'une communauté qu'incarne le locuteur natif des pays anglophones est pris par de nombreux enseignants et chercheurs comme un modèle de compétence linguistique à atteindre. Pourtant, en ce qui concerne les échanges en anglais lingua franca , cette conception résiste mal à la réalité du terrain car, comme les deux sections précédentes tendent à le montrer, les enjeux de la communication dans ce type de situation se situent à des niveaux davantage globaux.

Une preuve de ce défaut de résistance se trouve dans l'évaluation de niveau de compétence en langue réalisée sur le terrain par les professionnels eux-mêmes lors des entretiens d'embauche. Notre enquête de terrain montre, par exemple, que le directeur des ressources humaines (DRH) francophone d'une petite multinationale située en Alsace se fie assez peu aux résultats de tests de certification tel que le Test of English for International Communication (TOEIC), généralement fondés sur un modèle anglophone de la compétence, leur préférant l'entretien en anglais ou, comme ce fut le cas pour l'assistant RH dont une partie de la correspondance a été présentée dans cet article, la traduction d'une lettre portant sur un problème administratif précis. Manifestement donc, les locuteurs natifs ne sont plus les seuls à émettre des jugements d'acceptabilité. En ce qui concerne les échanges entre professionnels, la norme native atteindrait donc la limite du terrain où un nombre croissant de régularités, de mode de fonctionnement et autre tronc commun de caractéristiques lingua franca sont progressivement identifiés puis publiés.

27 La publication de caractéristiques phonétiques fondamentales en anglais lingua franca ( Lingua Franca Core) par J. Jenkins (2002) constituent un point de départ à l'identification de traits pouvant être considérés comme acceptables en situation d'échanges internationaux en même temps qu'elle est une contribution à la définition de l'acceptabilité dans ce contexte. Pour J.Jenkins, l'acceptabilité en contexte de communication internationale ne saurait être définie de manière exogène, c'est-à-dire par rapport aux normes des populations britannique (Received Pronunciation) et américaine (General American), mais de manière endogène, autrement dit par rapport à celles issues du cercle en expansion. 
Cette conception de la norme a conduit à un certain nombre d'avancées sur le plan lexicogrammatical. Parmi les publications plus récentes, notons la contribution d'Alessia Cogo et Martin Dewey (2012) qui, à partir du Vienna-Oxford Corpus of International English (VOICE), définissent un ensemble de traits lexico-grammaticaux pouvant être considérés comme certes erronés vis-à-vis des normes anglo-américaines mais acceptables d'un point de vue endogène. Tel est le cas de l'absence $d u$ « $s$ " à la troisième personne du singulier qui, selon les auteurs, est devenue l'option « par défaut dans les communication informelles et naturelles ${ }^{4}$ ( 2012 , p. 49). Il en est de même pour l'usage des prépositions ("I think it depends of the issue "; " we're just discussing about Japanese soup ", " we have the solution about this pollution ») qui, bien qu'elles ne respectent les normes exogènes, sont statistiquement fréquentes et n'entraînent pas de problèmes de communication.

Comme son nom l'indique, le corpus VOICE, sur lequel les résultats que nous venons d'aborder brièvement se fondent, est un corpus d'anglais oral où les enjeux de la communication sont, d'après le tableau 1, adapté de Language and the Internet (Crystal, 2001), sensiblement différents des échanges écrits.

Tableau 1. - Différences entre l'oral et l'écrit.

\begin{tabular}{|l|l|}
\hline Oral & Écrit \\
\hline - Immédiateté des échanges. & $\begin{array}{l}\text { •Caractère statique et permanent des } \\
\text { échanges. } \\
\text { - Production et réception simultannées. Quasi- } \\
\text { impossibilité de planifier. } \\
\text { - Erreurs non effaçables. }\end{array}$ \\
\hline & $\begin{array}{l}\text { Possibilité de planifier. } \\
\text { - Erreurs effaçables. }\end{array}$ \\
\hline
\end{tabular}

D'APRÈs D. CRYSTAL (2001, P. 26-27, ADAPTÉ).

Le caractère faiblement planifié de l'oral et fortement planifé de l'écrit engendre des erreurs par rapport à l'anglais standard moins fréquentes et de types différents. L'erreur emblématique de l'absence $\mathrm{du}$ 《 $\mathrm{s}$ » à la troisième personne du singulier est, par exemple, absente de notre corpus d'échanges de courriels, les scripteurs optant pour la grammaire standard sur ce point. Il en va de même pour le verbe "discuss" dont la plupart des occurrences dans notre corpus sont conformes à la norme anglo-américaine. En revanche, nos travaux sur la structure et la détermination des groupes nominaux mettent en évidence des écarts par rapport à la norme anglo-américaine. Nous en présentons ici quelques exemples :

Tableau 2. - Régularités d'emploi dans les articles et les groupes nominaux dans les échanges de courriels en anglais lingua franca professionnelle.

\begin{tabular}{|l|l|}
\hline $\begin{array}{l}\text { Emploi de l'article «the» avec des } \\
\text { données chiffrées. }\end{array}$ & $\begin{array}{l}\cdot \text { We would suggest to come in Okaszakki around the } \\
\text { week } \underline{20} . \\
\cdot \text { Let's start the test } \underline{2} . \\
\cdot \text { We will send you the invoices mentioning the tarif } \\
\text { number } \underline{7988,90} \text { as you requested. }\end{array}$ \\
\hline
\end{tabular}




\begin{tabular}{|l|l|}
\hline \multirow{2}{*}{$\begin{array}{l}\text { Augmentation du rôle du qualifieur } \\
\text { dans le groupe nominal. }\end{array}$} & $\begin{array}{l}\text { The part } \mathrm{A} / 3 \text { of the procedure. (norme anglo- } \\
\text { américaine : The } \mathrm{A} / 3 \text { part of the procedure). } \\
\text { The engine } 16 \mathrm{BM} 12 \text { (norme anglo-américaine : the } \\
16 \mathrm{BM} 12 \text { engine). } \\
\text { The coupling serie 52 (norme anglo-américaine : the } \\
\text { series 52 coupling). }\end{array}$ \\
\hline
\end{tabular}

Il convient d'ajouter à cela une tendance à la disparition de "that » au profit de «this ", employé quasi-universellement. Ces écarts par rapport à la norme anglo-américaine sont relativement réguliers dans notre corpus et concernent toutes les situations professionnelles d'échange. Outre la raison statistique, le caractère acceptable de ces traits tient au cadre relativement informel des courriels où les informations sont échangées rapidement, souvent dans l'urgence, généralement au sein de petits réseaux de collaborateurs familiers les uns des autres. Une seconde raison tient au fait qu'une partie significative de notre corpus met en scène des participants de langue romane. L'écart par rapport à la norme, du moins au niveau du groupe nominal, ne saurait ainsi être considéré comme une erreur "fatale», mais comme une erreur «collatérale » dans la mesure où il est partagé par les participants.

\section{Implications pédagogiques}

Les trois critères d'acceptabilité que nous venons de présenter conduisent à une conception de la langue, en tant qu'objet d'enseignement, où la maîtrise du code « natif " n'est plus l'objectif ultime mais constitue l'un des aspects de l'apprentissage. Comme cet article le suggère, l'enjeu de cet enseignement ne consiste pas à atteindre la compétence du locuteur natif mais de former des utilisateurs efficaces de langue anglaise. Notons que cette conception n'est pas nouvelle et qu'elle s'inscrit dans la lignée de tentatives relativement anciennes telles que l'anglais «BASIC» de Charles Odgen (1930) ou le " Nuclear English» de Randolph Quirk (1981). Toutefois, alors que ces tentatives présentent l'avantage d'avoir permis l'identification de traits lexico-grammaticaux fondamentaux pour la communication internationale, leur caractère simplificateur présente aujourd'hui un certain décalage au regard de la réalité complexe des milieux professionnels où, comme nous l'avons vu, l'expansion de l'anglais a conduit à l'augmentation de la variété des situations linguistiques, elle-même conduisant à la spécialisation accrue des échanges professionnels.

En dépit de cette évolution où les situations lingua franca occupent une place significative, force est de constater que l'anglais natif reste l'idéal à atteindre, comme en attestent ces extraits de la grille d'auto-évaluation issue du Cadre européen commun de référence (Conseil de l'Europe, 2000) où les auteurs invitent clairement les apprenants à maittriser les expressions idiomatiques et familières (sous entendu anglo-américaines), à accéder à la rapidité d'élocution des natifs, l'objectif étant d'interagir, dès le niveau B2, avec ces mêmes locuteurs.

Exemple 9. Extraits du Cadre européen commun de référence pour les langues

(Conseil de l'Europe, 2000, passages mis en gras par l'auteur). 
Je peux participer sans effort à toute conversation ou discussion et je suis aussi très à l'aise avec les expressions idiomatiques et les tournures courantes. (Interaction orale - C2)

Je peux communiquer avec un degré de spontanéité et d'aisance qui rende possible une interaction normale avec un locuteur natif. (Interaction orale - B2)

34 le fait que l'anglais possède la particularité d'être une, sinon « la » lingua franca mondiale. Cette contradiction prend d'autant plus d'ampleur lorsque l'on considère que le cadre est utilisé de manière extensive en Europe continentale, autrement dit hors Royaume-Uni, par des apprenants qui, pour la plupart sont inscrits dans les filières professionnalisantes, autrement dit, par de futurs utilisateurs de la langue anglaise tels que nous les avons définis précédemment. Il s'ensuit qu'un modèle pédagogique visant à former en anglais de futurs professionnels ne saurait se «limiter» à la seule acquisition d'un «Business English ", natif et orienté vers la culture des pays anglophones, mais sur un modèle plus large présentant l'anglais des situations non natives comme un objectif légitime.

La figure 4 (ci-dessous) est une tentative de représentation de ce modèle où l'anglais des milieux professionnels anglophones fusionne avec l'anglais lingua franca, la fusion donnant l'anglais lingua franca professionnelle. Dans ce cadre, le caractère acceptable ou inacceptable d'une proposition dépend moins de l'écart qu'elle présente par rapport à celle que produirait un locuteur fictif et idéal, que de son adéquation à la situation professionnelle et linguistique. En ce qui concerne l'axe professionnel, le caractère acceptable d'une proposition dépend de sa conformité aux genres et aux logiques professionnelles dans le cadre d'actions "spécialisées" au sens où elles tendent à émerger au sein d'échanges entre professionnels «experts». En cela, un cours de «langue professionnelle» est un cours de langue de spécialités visant à l'acquisition d'une culture professionnelle en général (apprendre à gérer les rapports sociaux en anglais, savoir accomplir des actions récurrentes à partir de genres transversaux) et d'une culture de spécialité (terminologie propre au métier visé, styles et logiques propres à chaque fonction et à chaque domaine spécialisé). 
Figure 4. - L'anglais comme lingua franca professionnelle : domaines et enjeux pédagogiques.

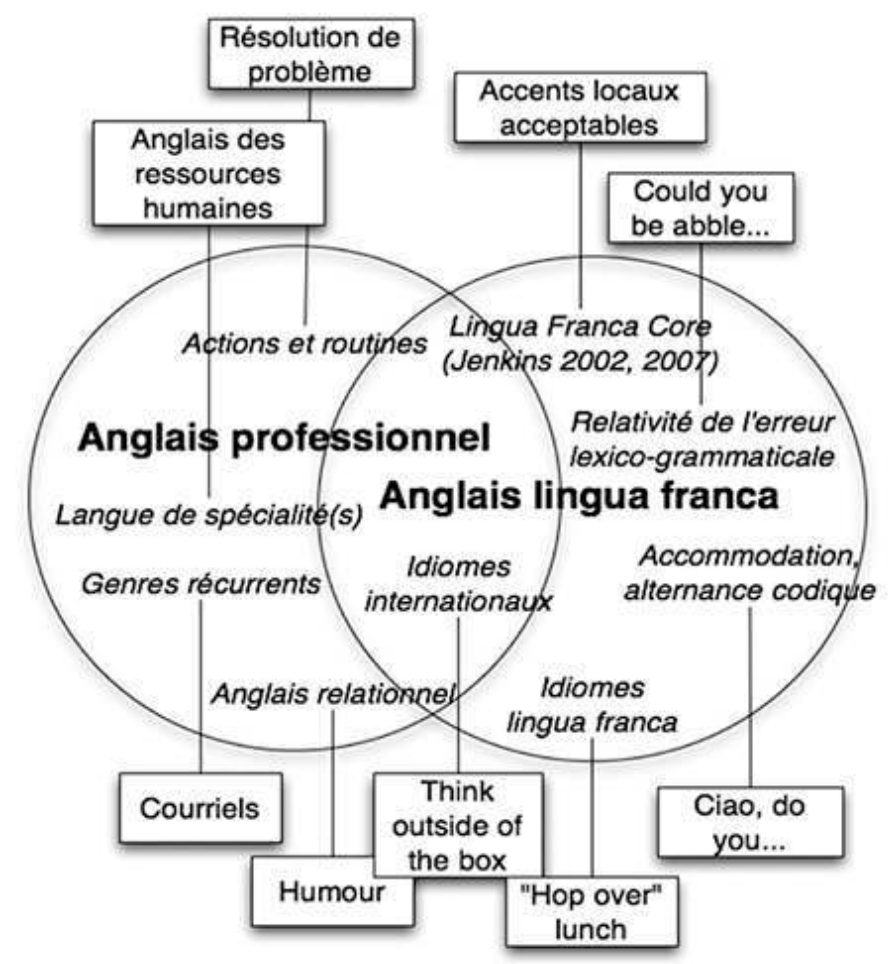

En ce qui concerne l'axe "lingua franca », l'acceptabilité dépend de l'accessibilité en termes phonétiques à partir du Lingua Franca Core et lexico-grammaticaux (limitation des erreurs fatales et tolérance à l'égard des erreurs collatérales). Dans ce cadre, la notion de situation, c'est-à-dire les paramètres de l'échange tels que le type d'action professionnelle en cours ou l'identité linguistique des locuteurs, occupe une place centrale dans l'évaluation et, pour ainsi dire, en détermine les critères. Cette approche suppose, par exemple, d'inciter les étudiants à utiliser des expressions idiomatiques anglo-américaines lorsqu'ils sont placés en interaction avec des natifs et, lorsqu'ils sont placés en situation d'échange lingua franca, de ne les utiliser que si elles peuvent être décodées à l'échelle internationale. Sur ce point, une expression telle que «think outside of the box " présente le double avantage d'être à la fois un idiome natif et une expression dont la force métaphorique est, nous semble-t-il, internationale.

\section{Conclusion}

L'acceptabilité est le point névralgique du débat que suscite l'apprentissage d'une variété qui, à l'heure actuelle, reste encore peu codifiée comparée à d'autres variétés telles que l'anglais standard. Peut-on - doit-on - enseigner une variété dont les réalisations lexicogrammaticales restent largement inacceptables au regard de l'anglais standard? Au vu de la recherche fertile dans ce domaine, tout porte à croire que la réponse soit affirmative. Comme notre article tend à le montrer, l'anglais standard (Trudgill \& Hannah, 2008) comme norme demeure relativement peu pertinent pour des professionnels davantage préoccupés par la réussite des actions professionnelles. Il s'avère en effet que, dans la plupart des situations observées, la conformité au code de l'anglais standard importe 
moins que la conformité aux fonctions communicatives telles que le genre et les logiques professionnelles et la capacité des participants à demeurer accessibles à partir d'un code malléable. Comme les exemples présentés le suggèrent cependant, ces trois critères ne sont pas exhaustifs et ne sauraient, à eux seuls, rendre les messages acceptables. L'acceptabilité relève au contraire d'une savante combinaison de critères discursifs, pragmatiques et lexico-grammaticaux opérée par des utilisateurs expérimentés de cette variété et permettant aux messages de fonctionner dans leur situation et, plus largement, dans le cadre de la culture professionnelle des employés. Nous comprenons alors qu'un enjeu essentiel dans la caractérisation d'une variété telle que l'anglais comme lingua franca professionnelle consiste à identifier puis modéliser des situations récurrentes d'échanges en vue de les rendre par la suite accessibles au public des apprenants. Sur ce point, les manuels d'anglais professionnels disponibles en France souffrent d'un manque de représentativité de la langue telle qu'elle est réellement pratiquée sur le terrain, le modèle étant à bien des égards fondé sur l'anglais standard. Comme ce qui précède le suggère, l'objectif d'une approche « lingua franca » dans ces manuels ne consisterait pas à « légaliser » aveuglément des comportements linguistiques déviants par rapport à la norme ni à l'envisager dans le cadre d'un manuel séparé, mais à indiquer, comme le font les grammairiens travaillant sur corpus depuis les années 1940, que dans certaines situations, certains comportements peuvent être considérés comme " acceptables » dans la mesure où la norme de l'anglais standard devient peu pertinente.

Enfin, soulignons que les acteurs principaux de la transmission de la langue, les enseignants, sont, du moins en France, relativement peu sensibilisés à la problématique de l'anglais utilisé comme lingua franca. Une piste explicative, que nous avançons ici, tiendrait à un problème d'adéquation entre les programmes dans les masters d'enseignement, où la linguistique de corpus, les World Englishes et l'anglais de spécialité sont largement sous-représentés, voire inexistants, et l'anglais tel qu'il est pratiqué sur le terrain. Pourtant, tout porte à croire que ces futurs acteurs enseigneront à des personnes - élèves de collèges et lycées, ou étudiants du secteur des Langues pour spécialistes des autres disciplines - dont on peut raisonnablement penser qu'elles seront, lors d'un séjour d'étude, d'un stage ou dans leur future carrière, confrontés à cette variété en expansion.

\section{BIBLIOGRAPHIE}

BIBER Douglas, JOHANSSON Stigg, LEECH Geoffrey, CONRAD Susan \& FINEGAN Edward, Longman Grammar of Spoken and Written English, Londres, Longman, 1999.

СНОмSку Noam, Syntactic Structures, Londres, Mouton, 1965.

Cogo Alessia, «ELF and Super-diversity. A Case Study of ELF Multilingual Practices from a Business Context », Journal of English as a Lingua Franca, vol. 1, n 4, 2012, p. 287-313.

COGO Alissa \& DEWEY Martin, Analysing English as a Lingua Franca. A Corpus-Driven Investigation, Londres, New York, Continuum, 2012.

CONSEIL de L'EUROPE, Cadre européen de référence pour les langues. Strasbourg, Didier, 2000. 
CRYSTAL David, English as a Global Language, Cambridge, Cambridge University Press, $2^{\mathrm{e}}$ édition, 2003.

-, Language and the Internet, Cambridge, Cambridge University Press, 2001.

FRIES Charles Carpenter, American English Grammar. The Grammatical Structure of Present-day American English with Especial Reference to Social Differences and Dialects, New York, D. AppletonCentury Company Incorporated, 1940.

GALlOWAY Nicola \& HEATH Rose, « They Envision Going to New York, Not Jakarta? The Differing Attitudes toward ELF of Students, Teaching Assistants, and Instructors in an English-Medium Business Program in Japan ", Journal of English as a Lingua Franca, vol. 2, n 2, 2013, p. 229-253. GRADDOL David, The Future of English, Londres, The British Institute, 2000.

HYMES Dell H, « On Communicative Competence », dans John Pride et Janet Holmes (éds), Sociolinguistics: Selected Readings, Harmondsworth, Penguins Books, 1972, p. 269-273.

ISANI Shaeda, "Compétence de culture professionnelle : définition, degrés et didactisation », ASp, vol. 43-44, 2004, p. 5-21.

JENKINS Jennifer, «A Sociolinguistically Based, Empirically Researched Pronunciation Syllabus for English as an International Language », Applied Linguistics, vol. 23, nº 1, 2002, p. 83-103.

-, English as a Lingua Franca. Attitude and Identity, Oxford, Oxford University Press, 2007.

-, World Englishes. A Resource Book for Students, Routledge, Londres, 2 ${ }^{\mathrm{e}}$ edition, 2009.

JENKINS Jennifer, MODIANO Marco \& SEIDLHOFER Barbara, « Euro-English », English Today, vol. 17, n 4, 2001, p. 13-9.

KACHRU Braj, «Les normes régionales de l'anglais », dans Édith Bédard et Jacques Maurais (éds), La norme linguistique, Québec, Gouvernement du Québec, Conseil de la langue française, 1983, p. 707-730.

-, «Standards, Codification, and Sociolinguistic Realism. The English Language in the Outer Circle ", dans Randolph Quirk et Henry Widdowson (éds), English in the World, Cambridge, Cambridge University Press, 1985, p. 11-30.

KANKAANRANTA Anna, Hej Seppo, Could You pls Comment on This! Internal Email Communication in Lingua Franca English in a Multinational Company, Jyväskylä, Jyväskylä university, 2005.

KIRKPATRICK Andrew, English as an International Language in Asia, Dordrecht, Springer, 2012. KOESTER Almut, Workplace Discourse, Londres, Continuum, 2010.

LABOV William, Sociolinguistics Patterns, Philadelphie, University of Pennsylvania Press, 1972. LEITNER Gerhard, « English as a Pluricentric Language », dans Michael Clyne (éd), Pluricentric Languages, Berlin, Mouton de Gruyter, 1992, p. 179-237.

MAURANEN Anna, HYNNINEN Nina \& RANTA Elina, « English as an Academic Lingua Franca: The ELFA Project », English for Specific Purposes, vol. 29, 2010, p. 183-190.

MOURLHON-DALLIES Françoise, Enseigner une langue à des fins professionnelles, Paris, Didier, 2008.

MILROY Leslie, Language and Social Networks, New York, Basil Blackwell, 2e édition, 1987.

NICKERSON Catherine, Playing the Corporate Language Game. An Investigation of the Genre and Discourse Strategies in English Used by Dutch Writers Working in Multinational Corporations, Amsterdam, Rodopi, 2000. 
ODGEN Charles, Basic English. A general introduction with rules and grammar, Londres, Kegan Paul, 1930.

QUIRK Randolph, «International Communication and the Concept of Nuclear English », dans Larry Smith, English for Cross-Cultural Communication, Londres, Macmillan, 1981, p. 151-165.

SEIDLHOFER Barbara, Understanding English as a Lingua Franca, Oxford, University Press, 2011.

SHAW Philip, « Conditions for Success in Lingua Franca Interaction », ASp, vol. 60, 2011, p. 65-79.

TRUDGILL Peter \& HANNAH Jean, International English. A Guide to the Varieties of Standard English, Londres, Hodder Education, $5^{\mathrm{e}}$ édition, 2008.

WANG Ying, « Non-Conformity to ENL Norms. A Perspective from Chinese English Users ", Journal of English as a Lingua Franca, vol. 2, n 2, 2013, p. 255-282.

WIDDOWSON Henry, « ELF and the Inconvenience of Established Concepts ", Journal of English as a

Lingua Franca, vol. 1, n 1, 2012, p. 5-26.

YANO Yasukata, « World Englishes in 2000 and Beyond », World Englishes, vol. 20, n 2, 2009,

p. 119-132.

\section{NOTES}

1. « Postman genre».

2. "An ideal speaker-listener in a completely homogeneous speech community, who knows his language perfectly» (Chomsky, 1965, p. 3).

3. "There is an important sense in which a normal member of a community has knowledge with respect to

[...] the communicative systems available to him. » (Hymes, 1972, p. 282)

4. « the default option in informal naturally occurring communcations » (Cogo \& Dewey, 2012, p. 49).

\section{RÉSUMÉS}

Le concept de locuteur-allocutaire idéal développé par Noam Chomksy (1965) et la place centrale qu'occupent les variétés natives de l'anglais sur la carte mentale du monde anglophone de Braj Kachru (1985) impliquent que la compétence en anglais devrait être calquée sur celle des locuteurs nés du "bon côté » de la carte et apprenant l'anglais «depuis le berceau ». Cette conception de la compétence est problématique dans les milieux professionnels où l'anglais est souvent une lingua franca, acquise par expérience dans le cadre spécialisé du métier des employés. Alors que les écarts par rapport à l'anglais dit «standard» sont fréquemment constatés et peuvent être considérés comme inacceptables vis-à-vis de la norme native, les études de terrain montrent qu'ils ne troublent pas le cours normal des actions. Il s'ensuit que, du moins dans le contexte professionnel, le modèle chomskyen de la compétence et la place centrale des natifs par rapport à la norme doivent être reconsidérés. Dans cet article, nous proposons un modèle de maîtrise de l'anglais comme lingua franca professionnelle fondé sur le concept d'utilisateur global et expérimenté d'une langue anglaise acquise au sein des réseaux socioprofessionnels internationaux. Ce modèle sert de point d'appui pour l'identification de trois 
critères d'acceptabilité qui, d'après notre observation des courriers électroniques professionnels, contribuent très largement à la réussite des interactions dans ce domaine. Enfin, cette réflexion nous amène à mesurer les incidences que peut avoir un tel modèle au niveau pédagogique où le niveau de compétence du locuteur natif reste, pour la plupart des institutions, l'objectif à atteindre.

The ideal speaker-listener concept developed by Noam Chomsky (1965) and the central position given to the native varieties of English on Braj Kachru's mental map of the English-speaking world largely imply that a competence model in English should derive from the speakers who were born on the "right side" of the map and who learnt English "from the cradle". However, as far as workplace interactions are concerned, the very notion of competence is problematic since English is very often a lingua franca acquired in the course of specialised, professional actions. Although many non-standard usages may be observed and considered unacceptable according to the native norms, field work increasingly shows that they do not impede the normal course of actions. This means that the chomskyan competence model and the central position occupied by native speakers should be reconsidered, at least as far as workplace interactions are concerned. In this article, we introduce a proficiency model based on global, experienced users of the English language involved in international, socio-professional networks. We use this model to identify three acceptability criteria which, according to our observation of professional emails, largely contribute to the success of interactions in this type of situation. Such a paradigm shift naturally leads us to consider some pedagogical implications for French institutions where native skills still form the basis of the teaching framework.

\section{INDEX}

Mots-clés : anglais comme lingua franca professionnelle, anglais standard, compétence linguistique, maîtrise de l'anglais, utilisateur global et expérimenté de la langue, genres, logiques professionnelles, courrier électronique

Keywords : English as a professional lingua franca, Standard English, linguistic competence, language proficiency, global experienced user of the English language, genres, professional reasoning, email

\section{AUTEUR}

\section{PHILIPPE MILLOT}

Université de Franche-Comté (France), ILCEA 